Editorial

\title{
Acknowledgement to Reviewers of Drones in 2018
}

\author{
Drones Editorial Office \\ MDPI, St. Alban-Anlage 66, 4052 Basel, Switzerland \\ Published: 8 January 2019
}

Rigorous peer-review is the corner-stone of high-quality academic publishing. The editorial team greatly appreciates the reviewers who contributed their knowledge and expertise to the journal's editorial process over the past 12 months. In 2018, a total of 45 papers were published in the journal, with a median time to first decision of 15 days and a median time to publication of 41 days. The editors would like to express their sincere gratitude to the following reviewers for their cooperation and dedication in 2018:

Abbes, Yosra

Abdelkefi, Abdessattar

Abdollahnejad, Azadeh

Aboutalebi, Mahyar

Adamo, Francesco

Adão, Telmo

Agapiou, Athos

Ahmad, Aamir

Alcarria, Ramon

Aldhaher, Samer

Al-Dunainawi, Yousif

Alsadik, Bashar

Alvear A., Oscar

Andrew, Margaret

Arza-García, Marcos

Baena, Susana

Bazi, Yakoub

Becker, Leandro Buss

Bento, Virgílio A.

Bilal, Muhammad

Bondi, Elizabeth

Brisson-Curadeau, Émile

Brito, Alisson

Burian, Adina

Calders, Kim

Cao, Chang

Carlucci, Antonio Paolo

Cavalli, Rosa

Chaurasia, Gaurav

Chen, Fuyang

Choudhary, Gaurav

Chrpa, Lukas

Clapuyt, François

Collins, Michael D.

Coombes, Matthew
Cowley, Dave

Cruz, Henry

Cueva, Román Alcides Lara

Cui, Rongxin

Culver, R. Lee

Dames, Philip

D'Andreagiovanni, Fabio

Davids, Corine

Deruyck, Margot

DEVEERASETTY, KRANTHI KUMAR

Di Sarli, Valeria

Domingues Franceschini, Marston Héracles

Duarte-Carvajalino, Julio M.

Dunn, James

Eekhout, Joris

Elbert, Philipp

Evangelatos, Orestis

Fass, Yehuda

Fletcher, Andrew

Flores, Gerardo

Forlani, Gianfranco

Frey, Julian

Gallacher, David

Gautam, Deepak

Giagkos, Alexandros

Głowacki, Paweł

Goel, Salil

Goes, Luiz Carlos Sandoval

Gojda, Martin

Gonçalves, José Alberto

González, Xesús P.

Grenzdörffer, Görres

Grimaccia, Francesco

Guerrero-Castellanos, José Fermi

Guida, Domenico 
Guo, Dejun

Habel, Jan Christian

Hashemi Beni, Leila

Hassanalian, Mostafa

He, Fangning

Heikkonen, Jukka

Herrick, Christina Czarnecki

Hesse, Henrik

Hession, Cully

Hicks, Thurston Cleveland

Hidalgo, Miguel Ángel Moreno

Hill, Geoff

Hoffmeister, Dirk

Hooper, Peter

Hortobágyi, Borbála

Hrishikeshavan, Vikram

Hsu, Hwai-Jung

$\mathrm{Hu}$, Qingwu

Hurford, Clive

Ilehag, Rebecca

James, Michael

Jia, Boru

Jiang, Mingjun

Jo, Hudyjaya Siswoyo

Joalland, Samuel

Joe, Woong Yeol

Joubert, Niels

Jung, Sunghun

Karas, Jakub

Koc, Ali Bulent

Kohv, Marko

Kreimer, Joseph

Kumar, Rajesh

Kweka, Bruno Joachim

La Cour-Harbo, Anders

Lai, Ying-Chih

Lamparelli, Rubens Augusto Camargo

Lauriks, Leen

Lee, Sungchang

Legare, Bryan

Lehmann, Jan R. K.

Levin, Eugen

Levy, Deborah

Li, $\mathrm{Na}$

Liebisch, Frank

Liu, Tao

Luís Marques Macato, André

Ly, Ngoc Q.

MacEachern, Scott

Marabissi, Dania

Marcato, José

Martinez, Patricio
Martinez-Espejo Zaragoza, Isabel

Mavropoulos, Georgios

Mesas Carrascosa, Francisco Javier

Miller, Boris

Moat, Justin F.

Mohan, Midhun

Montalvo, Carlos

Mostafa, Tarek M.

Mott, Rowan

Mozaffari, Mohammad

Mukhopadhyay, Shayok

Murtiyoso, Arnadi

$\mathrm{Na}$, Wongi S

Nansai, Shunsuke

Nevalainen, Olli

Oh, Hyongdong

Ota, Tetsuji

Overhage, Sven

Padró, Joan-Cristian

Pádua, Luís

Papa, Umberto

Paranjape, Aditya

Paredes, Jose A.

Partsinevelos, Panagiotis

Passalis, Nikolaos

Patil, Madhav

Perera, Asanka G.

Peres, Emanuel

Perroy, Ryan L.

PHAM, Quoc-Viet

Piancastelli, Luca

Poursanidis, Dimitris

Previtali, Mattia

Proctor, Cameron

Puente, Santiago

Ramon Soria, Pablo

Rana, Prashant S.

Reina, Daniel Gutiérrez

Resnik, David B.

Rhi, Seok-ho

Rodríguez-Vázquez, Fco. Javier

Roldán Gómez, Juan Jesús

Roscher, Ribana

Royo, Pablo

Rupnik, Ewelina

Salamí, Esther

Samiappan, Sathishkumar

Sanders, Abigail

SAVIN, IGOR

Schiano, Fabrizio

Schofield, Gail

Schreiber, Lance 
Seddon, Philip J.

Sha, Zongyao

Sharma, Vishal

Shults, Roman

Shutler, Jamie D.

Sirohi, Jayant

Skarlatos, Dimitrios

Song, Ziyou

Sonnemann, Till F.

Stamenkovic, Zoran

Stastny, Thomas

Stöcker, Claudia

$\mathrm{Su}$, Jinya

Sun, KeXun

Sutin, Alexander

Thiel, Christian

Ting Goh, Shu

Tong, Jinwu

Tsiropoulou, Eirini Eleni

Uddin, Mohammad Ammad

Vacca, Giuseppina

Verdonck, Lieven
Verstraete, Dries

Voit, Michael

Wakeham, Ronald

Wang, Xinyu

Weber, Konradin

Wei, Ping

Wen, Chih-Yu

Werden, Leland K.

Williamson, Liz

Witczak, Marcin

Xie, PU

Yakimenko, Oleg

Yang, Ming-Der

Yang, Tao

Yu, Shengwei

Yu, Tianqi

Zeghlache, Samir

Zhao, Tiebiao

Zhao, Ji

Zhong, Xiangnan

Zhu, Bo

(C) 2019 by the authors. Submitted for possible open access publication under the

terms and conditions of the Creative Commons Attribution (CC-BY) license (http://creativecommons.org/licenses/by/4.0/). 